\title{
Conhecimento dos pacientes com Doença Arterial Obstrutiva Periférica: estudo qualitativo
}

\author{
Knowledge of patients with Peripheral Obstructive Artery \\ Disease: qualitative study
}

\author{
Tatiana Martins', Nádia Chiodelli Salum², Melissa Orlandi Honório Lock², \\ Lucia Nazareth Amante ${ }^{4}$, Juliana Balbinot Reis Girondi ${ }^{5}$, Luciara Fabiane Sebold ${ }^{6}$ \\ Universidade Federal de Santa Catarina. Florianópolis, Santa Catarina, Brasil. ORCID: 0000-0002-0954-5811. tatianamartinsrn@gmail.com \\ ${ }^{2}$ Universidade Federal de Santa Catarina. Florianópolis, Santa Catarina, Brasil. ORCID: 0000-0002-2624-6477. nchiodelli@gmail.com \\ 3Universidade Federal de Santa Catarina. Florianópolis, Santa Catarina, Brasil. ORCID: 0000-0003-0972-2053. melhonorio@hotmail.com \\ ${ }^{4}$ Autora para correspondência. Universidade Federal de Santa Catarina. Florianópolis, Santa Catarina, Brasil. \\ ORCID: 0000-0002-5440-2094. Iuciamante@gmail.com \\ 5Universidade Federal de Santa Catarina. Florianópolis, Santa Catarina, Brasil. ORCID: 0000-0002-3763-4176. juliana.balbinot@ufsc.br \\ ${ }^{6}$ Universidade Federal de Santa Catarina. Florianópolis, Santa Catarina, Brasil. ORCID: 0000-0002-5023-9058. fabiane.sebold@ufsc.br
}

\begin{abstract}
RESUMO | Estudo descritivo exploratório com abordagem qualitativa, que teve por objetivo identificar o conhecimento do paciente hospitalizado acerca da doença arterial obstrutiva periférica e suas necessidades de cuidado no domicílio. A coleta de dados ocorreu de outubro a novembro de 2016, através de entrevista semiestruturada com 13 pacientes internados para tratamento da doença arterial obstrutiva periférica em uma clínica cirúrgica de um Hospital Universitário do sul do Brasil. A análise dos dados foi realizada através da análise temática, onde emergiu as seguintes categorias: Hábitos de vida; O viver com Doença Arterial Obstrutiva Periférica; A compreensão do paciente enquanto portador da Doença Arterial Obstrutiva Periférica; As dificuldades enfrentadas no domicílio e A rede de apoio. Os dados apontam que os pacientes não têm hábitos de vida saudáveis e entendem a doença como algo passageiro e de início súbito, não estando, portanto conscientes dos fatores de risco e da sua condição crônica. Apresentam dificuldades em adquirir medicamentos e realizar os curativos na rede básica de saúde, adotando como rede de apoio os familiares. Conclui-se que os pacientes possuem pouco conhecimento acerca do seu diagnóstico o que contribui para o agravamento de sua condição de saúde.
\end{abstract}

PALAVRAS-CHAVE: Enfermagem. Educação em saúde. Doença arterial periférica. Alta hospitalar.

\begin{abstract}
This is a descriptive-exploratory study with a qualitative approach aiming to identify the knowledge of the hospitalized patients about peripheral arterial occlusive disease and their needs for homecare. Data collection occurred from October to November 2016, through a semi-structured interview with 13 patients hospitalized for treatment of peripheral occlusive arterial disease in a surgical clinic of a university hospital in southern Brazil. Data analysis was performed through thematic analysis, from which the following categories emerged: Life habits; The understanding of the patient that lives with peripheral occlusive artery disease; The difficulties they face at home and The support network. Data show that patients do not have healthy lifestyles and that they understand the disease as temporary and that it onsets suddenly, therefore not being aware of risk factors and its chronic conditions. They present difficulties in acquiring medicine and performing bandage in the public health network, hence having their relatives as a support network. It is concluded that the patients have little knowledge of their diagnosis, which contributes to the worsening of their health condition.
\end{abstract}

KEYWORDS: Nursing. Health education. Peripheral arterial disease. Patient discharge 


\section{Introdução}

A Doença Arterial Obstrutiva Periférica (DAOP) consiste no estreitamento da luz das artérias que irrigam os membros inferiores, ocasionando uma redução do fluxo sanguíneo aos tecidos. Essa patologia caracteriza-se como multifatorial e tem como principal causa a doença aterosclerótica (Torres, et. al., 2012; Olin et. al., 2016). Embora a DAOP comprometa diretamente as regiões periféricas, tal como os membros inferiores, estudos apontam que essa doença está associada a diferentes eventos cardiovasculares e cerebrovasculares, sendo considerado um alerta na prevenção de tais eventos (Santos et al., 2013). Segundo o Ministério da Saúde (2011), as doenças cardiovasculares são responsáveis por mais de 30\% dos óbitos no Brasil, fato esse que gera um importante impacto econômico ao Sistema Único de Saúde (SUS). Conforme Migowski, et al. (2009) os custos do SUS com o tratamento de tais doenças no ano 2000 foi de 395 milhões de reais e em cinco anos esses custos elevaram-se para 736 milhões de reais. É possível que fatores como o surgimento de novas tecnologias, o envelhecimento da população, o aumento das doenças crônicas e, ainda, uma assistência à saúde voltada às doenças agudas estejam relacionados com o aumento nos custos do tratamento dessas doenças.

Nesse contexto, é possível apontar a DAOP como um problema da população contemporânea brasileira, uma vez que a sua prevenção consiste no controle dos fatores de risco, de caráter crônico, para o seu desenvolvimento e evolução. Pesquisas apontam como principais fatores de risco para a DAOP a Hipertensão Arterial Sistêmica (HAS), Diabetes Mellitus (DM), hiperlipidemia, obesidade e o tabagismo (Diniz; Pires, 2010; Lopes et al., 2012; Santos et al.,2013). Embora essa doença, em estágios avançados, possa levar a mutilações e à morte, se diagnosticada precocemente, pode ser tratada clinicamente sem a necessidade de intervenções cirúrgicas.

Para tal, é necessário que o paciente seja orientado acerca de sua problemática, visando esclarecê-lo sobre a doença, a sua evolução e a terapêutica proposta (Reis, et al., 2012). É visto que, a cronicidade da doença, associada a não adesão à terapêutica prescrita ocasiona o agravamento do quadro clínico do paciente, levando-o, muitas vezes, a mutilações físicas que comprometem negativamente a sua qualidade de vida (Vaz et al., 2013).
Essa perspectiva leva-nos a refletir sobre a prevenção de novas complicações como principal arma no controle das doenças, sobretudo a DAOP, sendo o autocuidado uma etapa indispensável nesse processo. Para tal, é fundamental que a educação em saúde, junto ao paciente hospitalizado, seja uma realidade dentro da esfera terciária de assistência à saúde, pois a falta de conhecimento acerca da doença perpetua os erros anteriormente cometidos, favorece a baixa adesão ao tratamento proposto e por fim leva a reinternações em piores condições de saúde (Teixeira, Rodrigues, Machado, 2012).

Nesse sentido, o complexo processo de educação pode ser entendido como o encontro entre os diferentes saberes, que une práticas do âmbito educacional e da saúde, com o conhecimento inerente ao indivíduo. Objetiva-se, através dessa fusão de conhecimento, uma aprendizagem significativa em que o saber do paciente representa o ponto de partida, visando, com isso, torná-lo o principal responsável de seu processo de saúde/doença e conscientizá-lo no que se refere aos cuidados necessários em prol de sua saúde, promovendo um agir consciente e autônomo (Marinho, Silva, 2015).

O processo de educação em saúde no âmbito hospitalar tem como um de seus objetivos principais, preparar o paciente para o retorno ao domicílio, de modo a torná-lo o principal responsável por sua recuperação e manutenção de seu bem-estar. Para isso, é necessário que a equipe multiprofissional perceba a alta hospitalar como um momento delicado, no qual existe uma complexa transição do cuidado profissional para o cuidado domiciliar, havendo, nesse momento, uma fusão de sentimentos, tais como: medo, ansiedade, decepção, contentamento (Reis, et al., 2013).

Isso posto, tem-se a contrareferência como uma importante etapa do planejamento da alta hospitalar, pois a comunicação entre os diferentes níveis de assistência à saúde é indispensável, de modo que exista não somente a troca de informações, mas também a sua perpetuação. A atuação da esfera primária junto ao paciente após a alta hospitalar é essencial no caso das condições crônicas como a DAOP, uma vez que essas doenças necessitam de tratamento contínuo e mudanças no estilo de vida (Caiafa, et al., 2011). 
Nessas condições, a comunicação entre os diferentes níveis de assistência à saúde possibilita uma meIhor continuidade do cuidado no que se refere ao retorno do paciente à comunidade, refletindo, também, na adesão do paciente a terapêutica proposta durante a hospitalização (Serra; Rodrigues, 2010). Configurando-se, assim, como uma rede de apoio ao paciente que prevê a articulação de ações de caráter curativo, preventivo, individuais ou coletivo que atendem às necessidades sociais em saúde. Para preparar este paciente para retornar ao domicílio é necessário identificar o quanto ele conhece de sua doença e tratamento e as demandas que repercutem na e reorganização de seus hábitos de vida para enfrentar os desafios frente a cronicidade da doença.

Nesse sentido, o estudo tem como questão norteadora: Qual o conhecimento do paciente acerca da DAOP e seu tratamento? Assim, levando-se em consideração que a necessidade de preparo do paciente para a alta hospitalar constitui um campo de estudo relevante, tem-se como objetivo identificar o conhecimento do paciente hospitalizado acerca da doença arterial obstrutiva periférica e suas necessidades de cuidado no domicílio.

\section{Método}

Trata-se de um estudo qualitativo, de caráter exploratório descritivo. Na abordagem qualitativa, a verdade é considerada um conjunto de realidades e contempla a complexidade do ser humano, utilizando-se para tal o olhar holístico na busca da percepção do todo (Polit e Beck, 2011).

O estudo foi realizado na Unidade de Clínica Cirúrgica de um Hospital Universitário do sul do país, que se caracteriza como um hospital de médio porte, de alta complexidade, sendo referência para todo o Estado de Santa Catarina em cirurgia vascular. A Unidade de Clínica Cirúrgica II caracteriza-se como uma unidade mista e, portanto, atende pacientes de ambos os sexos, em tratamento clínico e/ou pré e pós-operatório de cirurgia vascular. O setor é composto por 30 leitos, sendo destinados 12 leitos para cirurgia vascular, quatro para cirurgia plástica, oito para proctologia e seis para urologia (Hospital Universitário, 2016).
O estudo teve como participantes 13 pacientes internados em tratamento clínico e/ou cirúrgico convencional ou endovascular por diagnóstico de DAOP. Os participantes foram selecionados através do censo dos pacientes que é impresso diariamente na unidade, sendo incluídos para o estudo os pacientes maiores de dezoito anos; de ambos os sexos; com diagnóstico de DAOP; orientados em tempo e espaço. O critério de exclusão foi definido como: pacientes portadores de DAOP, porém internados para tratamento de outra comorbidade. Os dados foram coletados por meio de entrevista, realizada de outubro a novembro de 2016. Os pacientes foram convidados a participar durante a visita diária do enfermeiro (pesquisadora). Assim foi determinado uma data e local específico para realização da entrevista, a qual foi a sala de passagem de plantão da unidade. Foi elaborado um instrumento composto de perguntas abertas e fechadas que perpassa três momentos distintos: na Parte I (cinco perguntas fechadas e três perguntas abertas) realizou-se a investigação de: idade, sexo, estado civil, se reside com acompanhante, se possui alguém para auxiliar no cuidado, escolaridade, atividade laborativa, profissão, problemas de saúde e uso de medicamentos; na Parte II (quatro perguntas abertas) foram abordados os hábitos de vida (fumo, prática de exercícios físicos, alimentação) e na parte III (oito perguntas abertas) foram enfocados aspectos relacionados ao Diagnóstico e Tratamento (relato da história pregressa e atual, se houve uma explicação sobre sua doença de um profissional de saúde e como o paciente entende o seu problema, quais os cuidados que ele tem em casa, investigando a presença de uma pessoa para lhe ajudar e como é a ajuda, quais as facilidades e dificuldades para realizar o tratamento em casa).

A entrevista foi gravada e realizada conforme agendamento prévio com o paciente. A média de duração das entrevistas foi de 20 minutos, variando de acordo com a maneira de cada participante se expressar diante dos questionamentos. Os dados foram analisados a luz da análise temática, que consiste na execução de três importantes fases, definidas como: pré-análise; exploração do material e tratamento dos resultados e interpretação (Minayo, 2013). 
Na fase de pré-análise as entrevistas foram transcritas possibilitando uma leitura minuciosa e a organização do material por similaridade. Buscou-se, durante essa fase, identificar o conhecimento dos pacientes acerca da DAOP, os seus hábitos de vida, as dificuldades encontradas no domicílio após a alta hospitalar e as orientações necessárias para a continuidade do tratamento proposto após o seu retorno à comunidade. Na fase de exploração do material e tratamento dos resultados, o material obtido através das entrevistas foi interpretado de maneira fiel e divididas em pré-categorias. Por fim, na fase de tratamento dos resultados e interpretação, os dados foram separados em categorias temáticas que foram sustentadas e discutidas de acordo a literatura atual.

O Quadro 1 apresenta as partes do instrumento de pesquisa, o tipo de pergunta e as categorias que gerou.

Quadro 1. Demostrativo das categorias elencadas. Florianóplis, 2017

\begin{tabular}{|l|l|}
\hline Parte do instrumento de pesquisa/tipo de perguntas & Categoria que fundamentou \\
\hline Parte I (cinco perguntas fechadas e três abertas) & $\begin{array}{l}\text { Hábitos de vida do ser com doença arterial obstrutiva } \\
\text { periférica }\end{array}$ \\
\hline Parte II (quatro perguntas abertas) & $\begin{array}{l}\text { O conhecimento acerca da doença arterial obstrutiva } \\
\text { periférica }\end{array}$ \\
\hline Parte III (oito perguntas abertas) & $\begin{array}{l}\text { As dificuldades enfrentadas no domicílio } \\
\text { A rede de apoio }\end{array}$ \\
\hline
\end{tabular}

Fonte: As autoras, 2018.

O projeto foi submetido ao Comitê de Ética da UFSC (CEP/UFSC), conforme preconizado pela Resolução 466/12 do Conselho Nacional de Saúde, sobre a pesquisa envolvendo seres humanos, sendo aprovado pelo parecer $\mathrm{n}$. 1.744.247 do CEP/UFSC (CAAE 56958216.3.0000.0121). A aceitação dos pacientes foi obtida por meio da assinatura do Termo de Consentimento Livre e Esclarecido (TCLE). O anonimato foi mantido utilizando-se identificação alfanumérica ( $P$ - pacientes ou AP- acompanhante do paciente, seguido de uma ordem numérica de 1 a 13).

\section{Resultados}

Os nove participantes do estudo encontravam-se internados na Unidade de Clínica Cirúrgica no momento da entrevista, com idade entre 46 a 78 anos, sendo um paciente com idade entre 40-50 anos, dois entre 51-60 anos, seis entre 61-70 anos e quatro com idade entre 71-80 anos. Dos 13 pacientes entrevistados sete são do sexo feminino e seis do sexo masculino. Quanto ao estado civil, quatro encontravam-se casados no momento da entrevista, dois em uma união estável, cinco viúvos e dois divorciados. Dos entrevistados, 12 têm filhos e apenas um não tem, sendo que 11 residem na companhia dos cônjuges, filhos ou familiares e apenas dois residem sozinhos. Em relação à escolaridade, dois possuíam o $2^{\circ}$ grau completo, três o ensino fundamental completo e oito o ensino fundamental incompleto. Desse modo, a baixa escolaridade mostrou-se predominante entre os participantes do estudo, uma vez que de sete desses não concluíram o ensino fundamental. Em relação à atividade laboral um paciente informou estar desempregado, um ser pensionista e duas mulheres definiram-se como donas de casa e, portanto, dependem financeiramente de seus cônjuges. Sete dos participantes são aposentados e dois relataram que trabalham, contudo, encontram-se afastados de suas atividades laborais por licença médica para tratamento de saúde. 
Através da ordenação e reflexão dos dados, emergiram as seguintes categorias: Hábitos de vida do ser com doença arterial obstrutiva periférica; Viver com doença arterial obstrutiva periférica; $O$ conhecimento acerca da doença arterial obstrutiva periférica; As dificuldades enfrentadas no domicílio; A rede de apoio.

\section{Hábitos de vida do ser com doença arterial obstrutiva periférica}

Nessa categoria foram organizados os relatos que contemplam os hábitos de vida dos pacientes com diagnóstico de DAOP, sendo contempladas questões relacionadas com o hábito de fumar, prática de atividades físicas e hábitos alimentares. Em relação ao hábito do tabagismo, dois pacientes afirmaram que fumam, quatro nunca fumaram e sete são ex-tabagistas. No que se refere aos hábitos de vida, dois hábitos não saudáveis e fortemente relacionados com a DAOP mostraram-se presentes: o tabagismo e o sedentarismo.

\section{Já fumei muito, mas agora não [...] Fumava duas carteiras de cigarro por dia (P2)}

Fumava 20 cigarros por dia antes de me internar (P3).

Parei de fumar há 25 anos. Fumava duas a três carteiras por dia [...] hoje eu estou com 68. Comecei a fumar com 16 anos (P13).

No que se refere à prática de atividades físicas, nove participantes reconheceram-se como sedentários e relataram não praticar nenhum exercício físico; três informaram que caminhavam com frequência antes de ficarem doentes e um participante informou que jogava futebol uma vez na semana antes da internação hospitalar.

Não [pratico atividade física]. Há mais de 30 anos jogava futebol umas três vezes na semana (P2).

Nunca pratiquei (P3). Eu andava bastante. Agora que eu não estou caminhando mais (P13).

Quanto aos hábitos alimentares, os 13 participantes demonstram ter conhecimento sobre os cuidados que devem manter com a alimentação, porém apesar de conhecerem os malefícios de alguns alimentos relataramquenãofazemnenhumarestriçãoalimentar. Questões de ordem financeira foram informadas pelos pacientes entrevistados como um obstáculo na adesão à dieta recomendada.

Então, eu já tentei várias vezes fazer uma dieta especifica, mas não cheguei ao fim (P13).

Na hora que dava vontade de comer, comia (P10).

Eu já tentei fazer algumas vezes [fazer uma dieta controlada], mas baixa demais a diabetes e eu passo mal. Então eu já tentei várias vezes fazer uma dieta específica, mas não cheguei ao fim (P9).

Ela estava tomando leite desnatado, mas daí acaba e não dá para comprar e ela acaba voltando para o integral (AP5).

\section{Viver com doença arterial obstrutiva periférica}

Nessa categoria foram contemplados os relatos que fazem alusão às comorbidades associadas ao desenvolvimento e evolução da DAOP.

Foram abordadas questões relacionadas com as comorbidades de HAS e DM. A associação da HAS com o DM foi relatada por 12 dos entrevistados. Estes informaram que utilizam diariamente medicamentos como: anti-hipertensivos, hipoglicemiantes, insulina ou a associação entre essas terapias farmacológicas. Um participante informou ser diabético e utilizar, além dos medicamentos prescritos para o tratamento do DM, fármacos para doenças no coração, entretanto não soube informar se era hipertenso.

Uso todo remédio que o diabético tem que tomar, eu uso. Insulina, comprimido, tudo [...] tenho pressão [alta], mas tomo remédio para pressão, a minha pressão é 13, é 12. (P10)

Para diabetes eu tomo insulina regular e tomo comprimido. Tomo dois comprimidos e quando tem eu tomo três, que é para não afetar o coração. Aquele infantil, o AAS. Para tudo eu tomo medicamento (P12).

Embora o número de hipertensos e/ou diabéticos seja elevado, muitos pacientes comunicaram que não fazem acompanhamento com frequência de tais comorbidades e se limitam a terapia medicamentosa prescrita que, muitas vezes, encontra-se desatualizada frente a evolução da doença. 
Não, não acompanho [a pressão arterial e o DM no

Posto de Saúde]. Eu voltei lá na sexta-feira, depois de um ano, para ver a pressão e a diabetes. E estava alta, a pressão e a diabetes (P6).

Nessas condições, é importante ressaltar que a HAS e o DM encontram-se entre os fatores de risco mais comuns para o surgimento e progressão da DAOP e, portanto, se não tratados corretamente contribuem com a evolução da DAOP para casos clinicamente mais graves.

\section{O conhecimento acerca da doença arterial obs- trutiva periférica}

Compreender a DAOP é o primeiro passo para se alcançar o êxito do tratamento proposto. Entender a cronicidade da doença, os fatores que levaram ao adoecimento e as alternativas para controlar a evolução da doença representam elementos essenciais para a promoção da saúde e manutenção do bem-estar do paciente. Contudo, tem-se entre a baixa escolaridade da população estudada como um fator dificultador desse processo. Fato que requer dos profissionais de saúde uma dedicação especial para se fazer entender. Observou-se, a partir das entrevistas, que muitos dos pacientes hospitalizados para tratamento da DAOP, desconhecem tanto seu diagnóstico médico, quanto os motivos que levaram ao seu adoecimento. Embora alguns profissionais de saúde tenham explicado sobre a DAOP e o seu tratamento, as informações discutidas foram pouco esclarecedoras e assimiladas. Quando questionados como entendiam a DAOP, as falas apontam:

Do joelho para baixo as veias são muito fininhas, é onde não tem a passagem. Foi o que eu entendi (P1).

O que eles explicaram hoje é que está infeccionado. Que a infecção já tomou conta das veias ali e por isso que eles optaram pela amputação de coxa que vai ser

feita amanhã. Porque já está bem infeccionado e a infecção já está indo para o sangue. (AP3)

Não [me explicaram]. Uma explicação assim que a gente pudesse entender não. Eles não explicam assim. Eles dizem que é por causa do cigarro (P7).

Eu entendi que eu tenho diabetes, que eu tenho que me cuidar. Vai começar amputando, amputando até morrer (P12).
Embora os pacientes tenham informado que algum profissional de saúde tenha explicado acerca de sua doença, as informações não foram assimiladas devidamente e sete dos pacientes entrevistados associaram os acontecimentos ocasionais como o efeito de bater involuntariamente com o pé em um objeto, como o principal motivo de seu adoecimento. O trauma pode propiciar a agudização da DAOP, entretanto não se configura como a etiologia da doença (Lopes et al., 2012). A esse propósito, os pacientes entrevistados que informaram ter compreendido a explicação sobre a sua doença, definiram o seu diagnóstico como um problema de circulação ou de falta de circulação nas pernas. Informaram ainda, que o diabetes foi o principal motivo para o seu adoecimento. Embora o diabetes, de fato, seja um dos principais fatores de risco para a DAOP, sabe-se que outros elementos foram determinantes no processo de adoecimento, tais como o tabagismo, a inatividade física, os elevados níveis pressóricos e a hiperlipidemia (Reis et. al., 2012; Rolim, et. al., 2015).

Contrapondo-se a necessidade de orientação, foi observado nas falas dos pacientes que a explicação dos profissionais de saúde no que tange ao diagnóstico médico mostra-se pouco esclarecedora, muito provavelmente pela utilização de termos técnicos ou palavras desconhecidas pelos pacientes com a DAOP.

Eles não falam declarado, sabe eles não falam assim:

Dona P1. a senhora teve isso e isso. Não, eles falam salteado, então você entenda o que quiser entender (P1).

Não caiu a minha ficha. Está passando mil coisas na minha cabeça que eu possa vir a perder a perna, qualquer coisa. Porque eu não consegui sentar com uma pessoa que pudesse me explicar mais ou menos como é que seria isso (P7).

\section{As dificuldades enfrentadas no domicílio}

A alta hospitalar configura-se como um momento crítico da hospitalização e, nessa transição, é essencial que o conhecimento do paciente no que tange ao seu diagnóstico e o controle dos fatores de risco, encontrem-se bem consolidados. Pois, do contrário, o agravamento da DAOP será uma realidade com o transcorrer do tempo. Desse modo, quando 
questionados sobre os cuidados com a DAOP no domicílio, nove pacientes relataram que possuem dificuldade quanto ao seu autocuidado, havendo uma maior preocupação, sobretudo, com a troca de curativo e o acesso aos medicamentos prescritos. Todavia, dois pacientes informaram não ter dificuldades na continuidade dos cuidados após a alta hospitalar em razão do apoio familiar no domicílio.

Vai ser o curativo, vou ver se faço um acerto com o posto de saúde que é perto da minha casa para ver se eles fazem durante a semana e no final de semana algum familiar irá fazer para mim (P4).

Minha dificuldade é a medicação, de não ter acesso, porque se você vai procurar no posto geralmente não tem. Entendeu? Geralmente não tem. Porque antes de vir para cá eu ia lá procurar remédio para dor e o remédio para dor geralmente não tinha (P1).

Existe uma consciência inerente do paciente no que diz respeito ao seu autocuidado, uma vez que demonstra entendimento quanto à necessidade em modificar alguns hábitos praticados em seu cotidiano. A necessidade de uma alimentação mais saudável dentro de suas possibilidades econômicas, o controle do peso, a cessação tabágica, a utilização correta dos medicamentos e o sedentarismo foram pontos comumente citados pelos pacientes quando questionados sobre os cuidados com a saúde que seriam necessários após a alta hospitalar.

Eu vou ter que mudar muita coisa na minha alimentação, eu vou ter que comer mais frutas, mais verduras para as células poderem se regenerarem (P1).

Vou ter que mudar a alimentação, que é primordial, exercício e cuidados maiores com saúde, pela minha idade né, 70 anos. (P4)

Um número expressivo dos pacientes entrevistados manifestou o desejo e a intenção de manter uma alimentação mais saudável ao retornar ao domicílio, contudo, informaram simultaneamente a esse desejo que possuem dificuldade em comprar os alimentos recomendados por questões de cunho financeiro. Quanto aos pacientes que moram sozinhos, o preparo dos alimentos também se configurou como um obstáculo, fato que propicia o consumo de alimentos inadequados.
Moro sozinho e não sei fazer nada, eu mal sei fazer um arrozinho. Verdura e coisa diet assim eu não me preocupo, quando vou ao supermercado não me preocupo com isso. Eu quero ver se arrumo uma pessoa, se ela não me cobrar muito caro. Aí eu vou pegar ela para fazer a comida especial (P2).

Alguns participantes do estudo expuseram a dificuldade de custear a dieta recomendada e o tratamento medicamentoso como um obstáculo à terapêutica prescrita. Relatam que em períodos de paralisação dos serviços públicos de saúde, tal como as greves, a terapia medicamentosa precisou ser suspensa até a normalização da assistência por parte desses órgãos.

Já tive 3 dias sem tomar insulina. E eu tomo insulina 22 (unidades) de manhã e 22 a noite. Se não tomar vai a 500,600, como uma vez eu vim parar aqui. Claro só tomei soro e voltei para casa, porque não tinha insulina, os postos estavam tudo em greve (P13).

Eles vão me receitar um tipo de remédio aqui para fazer o tratamento em casa, daí chega no postinho e o postinho não tem. Isso daí você que atua na área da saúde está por dentro. Então as maiores dificuldades do pobre é isso aí! É greve em cima de greve, quando abre o postinho não tem remédio. Quando tem insulina, não tem agulha e assim vai. Tem um tipo de remédio e não tem o outro (P12).

\section{A rede de apoio}

Esta categoria abrange a rede de apoio que o paciente utiliza para a continuidade do seu tratamento no domicílio, seja ela dos familiares, comunidade ou serviços públicos de saúde. O cuidado realizado por familiares após a alta hospitalar, representado pelo cônjuge, filhos e netos configurou-se como a principal rede de apoio entre os entrevistados. Tais cuidados perpassam desde o auxílio com os cuidados de higiene, realização de curativos, das atividades domésticas como alimentação e limpeza do domicílio até o acompanhamento aos serviços de saúde.

Meus filhos me ajudam na limpeza [da casa], nos curativos, na medicação. Fazem um pouquinho de tudo (P3).

Meu filho. Ele faz almoço, lava a roupa na máquina, ele faz tudo. São muitos os remédios que eu tomo. Então ele colocou tudo no papelzinho (P10). 
Logo, percebe-se que os pacientes com DAOP apresentam algum grau de dependência para a realização de suas atividades diárias, tais como: o banho, curativo, preparo de alimentos, administração dos medicamentos, entre outros. Fato que se justifica pela idade avançada e pela limitação física que a doença ocasiona à medida que evolui.

O mesmo foi exposto por Rolim et. al. (2015) que associaram o agrupamento de comorbidades e o envelhecimento da população como fatores que dificultam o processo de recuperação do paciente. Dessa forma, a presença dos filhos, a participação do cônjuge e demais familiares tem se mostrado um dos principais fatores no processo de recuperação do paciente após a alta hospitalar. Pois, aqueles que possuem uma boa estrutura familiar, encontram, nessa relação, um amparo para a realização das atividades do cotidiano.

Acho que eu não tenho dificuldade, porque eu tenho bastante gente para me ajudar, tem a filha, tem o neto, tem a esposa do meu neto (P5).

O que se observa é que o sistema de atenção básica de saúde foi pouco lembrado pelos pacientes como apoio para a continuidade do tratamento, de maneira que sua utilização permaneceu restrita ao fornecimento de medicamentos e materiais para realização dos curativos no domicílio. Entende-se, portanto, que a rede básica de saúde encontra-se subutilizada, de maneira que o seu potencial não está sendo explorado em toda sua plenitude.

Consulta [no posto de saúde] eu não confio muito não. Mas eu gosto das enfermeiras. Vou pegar as fitas que tem que ir naquelas reuniões. Eu ainda não tenho idade para ir em casa. Meu marido eles iam (P10).

Vamos pegar os materiais no posto de saúde e quem vai fazer o curativo é a minha cunhada (P8).

Sim, eu uso o postinho. Eu pego o remédio todo mês (P12).

\section{Discussão}

O objetivo desse estudo foi identificar o conhecimento do paciente hospitalizado acerca da DAOP e quais as necessidades de cuidado no domicílio. Para tal, conhecer o cotidiano dos pacientes, a sua história de vida pregressa e o seu modo de viver foram elementos fundamentais para essa construção.

Conhecer o nível de escolarização representou uma etapa indispensável e uma das grandes problemáticas identificadas, pois, embora saibamos que o conhecimento é inerente a todos e que a sabedoria é um bem adquirido no decurso do processo de viver, a escolaridade expressa pelos anos de estudo de um indivíduo denota importante papel nos seus hábitos de vida.

Em consonância com outros achados científicos, esse estudo apontou um baixo nível de escolaridade no que se refere aos participantes da pesquisa e portadores da DAOP, sendo a média escolar inferior a nove anos, o que corresponde ao ensino fundamental incompleto (Diniz; Pires, 2010; Reis et al., 2013).

Ratificando tais questões, uma pesquisa realizada com 54.369 indivíduos com idade igual ou maior a 18 anos nas capitais brasileiras e distrito federal, relacionou a baixa escolaridade com o hábito de fumar (Brasil, 2015).

Berto, Carvalhaes, Moura (2010) observaram que o tabagismo é maior entre os indivíduos de baixa escolaridade, sendo inversamente proporcional ao tempo de escola. Ou seja, quanto maior o período de atividade escolar, menor a prevalência desse hábito tão fortemente associado a diversas doenças, como o câncer, doenças cardiovasculares, cerebrovasculares e pulmonares. Este fato implica na adequação de estratégias de informação aceca dos malefícios do tabaco e de ferramentas de cessação disponíveis.

É possível inferir nesse caso, que o conhecimento adquirido através da escola representa um fator de proteção no que tange ao tabagismo. Foi visto, também, que a baixa escolaridade está diretamente relacionada a uma alimentação inadequada, ao sedentarismo e, por conseguinte, a obesidade. 
Em estudo como objetivo de investigar a prevalência de DAP e seus fatores de risco na população brasileira identificou como fatores de risco para DAP na população investigada o tabagismo, sedentarismo, diabetes mellitus e idade, que são semelhantes aos de outros estudos epidemiológicos incluindo diferentes grupos étnicos (Alvim, et al., 2018).

A esse respeito, Bastos et al. (2012) concluíram em um estudo realizado com 449 homens com idade entre 20 e 59 anos residentes em Campinas- São Paulo, que o menor período ou o tempo de atividade escolar, está associado a hábitos não saudáveis e, portanto, maior consumo de bebida alcoólica, sedentarismo, alimentação inadequada e tabagismo.

Malta e Menrhy (2010) relatam que a falta de ações que previnam o adoecimento da população e promovam a saúde do indivíduo, como a prática de atividade física, pode ser compreendida como uma falha no processo de gerenciamento do setor saúde. Pois, medidas de prevenção e promoção da saúde devem ser estimuladas a fim de evitar a entrada do indivíduo doente nas redes de serviços. Logo, a ausência do hábito de exercitar-se ou o sedentarismo representa um problema que se agrava a cada dia na sociedade contemporânea, é intrigante que esse hábito saudável, de baixo ou nenhum custo seja tão pouco explorado. Somado a essa realidade, a pesquisa identificou, também, elementos de grande complexidade quanto ao diagnóstico, tratamento e evolução da doença arterial periférica.

Em estudo realizado com 174 paciente para descrever os padrões de atividade física de pacientes com DOAP sintomática demonstrou que o padrão de atividade física de pacientes brasileiros com sintomas de claudicação intermitente é caracterizado pelo alto tempo gasto em comportamento sedentário e baixo envolvimento em atividades físicas, sendo que apenas 3,4\% desses pacientes atendem às recomendações atuais de atividade física para a população em geral (Gerage et al., 2019).

A questão socioeconômica emergiu como um elemento para a prevalência de fatores de risco para a DAOP, tais como o tabagismo, o sedentarismo e a alimentação inadequada com pouco consumo de frutas, verduras e hortaliças e elevada ingestão de frituras e refrigerantes (Markdisse et al., 2008; Sales et al., 2015; Alvin, 2018).
No que tange aos fatores de risco para o desenvolvimento da DAOP, os pacientes demonstraram desconhecer tais fatores, sobretudo a influência da HAS e do DM, haja vista muitos informaram que não acompanham seus níveis pressóricos, consideram seu diabetes de baixa intensidade e não realizam acompanhamento de tais comorbidades na rede básica de saúde. Em estudo realizado com o objetivo de avaliar as taxas de adesão a fármacos anti-hipertensivos de pacientes hipertensos identificou baixa adesão ao tratamento e que a maioria dos pacientes que relatam dificuldades em identificar os fármacos prescritos, pois não sabiam o nome do medicamento do qual faziam uso contínuo. Das principais razões para a não adesão medicamentosa, o esquecimento foi o maior motivo apontado, entre outros como o dos custos dos fármacos, a tolerância ao regime de fármacos, não uso de álcool (Oliveira et al., 2013).

Nessas condições, é importante ressaltar que a HAS e o DM encontram-se entre os fatores de risco mais comuns para o surgimento e progressão da DAOP e, portanto, se não tratados corretamente contribuem com a evolução da doença para casos clinicamente mais graves (Rolim et al., 2015).

Diante dessa perspectiva, foi identificada entre os participantes do estudo uma consciência, ainda que elementar da necessidade em adquirir hábitos de vida saudáveis, no intuito de controlar a doença. Entretanto, quanto ao diagnóstico médico, os pacientes demonstraram pouco conhecimento acerca de sua doença, pondo em evidência um conjunto de problemas com raízes profundas dentro da dinâmica hospitalar, na qual a velocidade com que os procedimentos são executados, a agilidade dos profissionais que ali atuam, bem como o predomínio das tecnologias duras, mostra-se como os grandes regentes do hospital. Nota-se uma necessidade em exaltar a importância da comunicação terapêutica, da empatia e da educação em saúde na alta complexidade, a fim de tornar o paciente o principal responsável pelo seu processo de saúde e doença, como também torná-lo capaz de decidir e discutir sobre o tratamento proposto. Acredita-se, de forma que a conscientização dos pacientes sobre seus riscos contribui para a melhor adesão a terapia proposta (Brondani et al., 2010, Oliveira, et al., 2013). 
Houve similaridade entre esse estudo e o resultado encontrado por Diniz e Pires (2010), uma vez que ambas as pesquisas obtiveram um percentual superior a $60 \%$ no que se refere aos pacientes com DAOP que desconheciam seu diagnóstico. Nessas condições, a DAOP foi interpretada por essa população como uma doença aguda, de surgimento súbito. O fato de compreender a doença como súbita, está relacionado com sua evolução silenciosa, o que transmite a falsa impressão de uma condição de início inesperado.

Desse modo, é essencial consolidar o conhecimento do paciente acerca de seu diagnóstico e, principalmente, da necessidade do controle dos fatores de risco da doença. Pois, do contrário o agravamento da DAOP será uma realidade com o transcorrer do tempo. Assim fazendo, o processo de educação em saúde durante a permanência no hospital configura-se como uma etapa vital para êxito do tratamento, todavia esse processo deve se estender a comunidade, devendo o conhecimento se renovar continuamente e adaptar-se diante da dinamicidade do viver de cada um.

A baixa escolaridade dos pacientes entrevistados representou um elemento de grande relevância no que se refere à compreensão de seu processo de saúde e doença, assim como das informações discutidas durante a internação hospitalar.

Diante de tais considerações, alguns questionamentos surgem perante o exposto: seria o baixo nível de instrução do paciente com DAOP o principal fator para o pouco conhecimento acerca de sua doença? Qual a responsabilidade dos profissionais de saúde defronte a essa problemática? O processo de educação em saúde não estaria sendo negligenciado pelos profissionais da área? Entende-se que o paciente deve ser o principal responsável pela sua recuperação e permanência de seu bem-estar, entretanto para que tal afirmação se faça verdade, é essencial que o poder de decisão esteja com o protagonista do cuidado. Mas, como decidir entre fumar ou abandonar o tabagismo? Entre exercitar-se ou manter-se sedentário? Ou ainda, o que comer? Como preparar os alimentos? O poder de decisão ou escolha torna-se inviável se o paciente desconhece os fatores que contribuíram com o seu adoecimento, existe uma grande possibilidade mediante esse desconhecimento que os maus hábitos de vida sejam mantidos.
Quanto às dificuldades enfrentadas no domicílio, a realização correta do curativo representa um ponto de grande preocupação dos pacientes após a alta hospitalar. Na realidade, a apreensão no que refere cicatrização da lesão é justificada pelo impacto que a ferida ocasiona na vida de cada paciente, uma vez que está associada a diversos componentes de ordem emocional.

A lesão trófica pode ser a causa de um afastamento social, conjugal, profissional ou, ainda, a associação de tais questões. $O$ fato é que a carga emocional de ser portador de uma ferida ou ter sido submetido a amputações seja de ordem maior ou menor, exige um novo olhar sobre si mesmo e necessita do amparo profissional para o enfrentamento dessa nova condição (Fonseca et al., 2012).

Destaca-se que o acompanhamento da lesão trófica por um profissional de saúde pode ser determinante no tratamento do paciente, pois tais feridas apresentam um processo de cicatrização longo, que exige uma avaliação diária quanto a sua evolução e características.

Entende-se que a troca do curativo representa um momento de grande importância, pois é a ocasião ideal para se avaliar os elementos que compõe o processo cicatricial, tais como: cor, odor, exsudação, tamanho e pele perilesional. A evolução da lesão trófica transcende o processo de restauração dos tecidos e denota, também, aspectos como o estado nutricional, a condição clínica do paciente e, ainda, o sucesso da terapêutica proposta. Portanto, o acompanhamento inadequado ou olhar inexperiente do cuidador diante da ferida podem propiciar o agravamento de uma condição de saúde ou postergar o processo de recuperação do paciente (Fonseca et al., 2012).

A rede de apoio ao paciente com DAOP apresentou-se como um diferencial após a alta hospitalar, pois muitos são os questionamentos, as dificuldades e incertezas frente ao diagnóstico da doença. Dessa forma, o apoio de familiares, amigos, profissionais e redes de assistência à saúde representa um elemento fundamental para o enfrentamento da doença (Tavares; Silva, 2013). 
O apoio familiar emergiu como um aspecto de grande relevância e um diferencial no que tange a continuidade do tratamento após a alta hospitalar, sendo o apoio da família um agente facilitador desse processo (Brondani et al., 2010). Tais resultados reafirmam a importância das relações sociais enquanto indivíduo do mundo.

Fonseca et al. (2012) e Barros et al. (2012) afirmam que os laços de afeto estabelecidos entre familiares, cuidadores e amigos favorece o processo de recuperação do paciente, estimula a sua autoestima e autocuidado. Assim, o paciente sente-se acolhido e emocionalmente mais fortalecido para enfrentar e adaptar-se a sua nova condição.

A aquisição dos medicamentos prescritos durante a internação hospitalar surgiu como um fator dificultador na adesão ao tratamento da doença, visto que nem todos os medicamentos prescritos são facilmente distribuídos pelo SUS, segundo os entrevistados. Por outro lado, quando existe greve no setor público de saúde, os medicamentos deixam de ser entregues, implicando na suspensão da terapia medicamentosa.

A dificuldade em compreender as orientações dos profissionais de saúde, como também o pouco esclarecimento no que tange ao seu diagnóstico e prognóstico da doença mostraram-se presentes. Desse modo, Diniz, Pires (2010) destaca a importância da utilização de uma linguagem clara com esse público, uma vez que os termos de cunho científico ou o uso de elementos linguísticos de grande complexidade dificultam o entendimento dos pacientes.

Nesse sentido, observa-se a educação em saúde como uma etapa primordial do processo de trabaIho dos profissionais de saúde, uma vez que tem a finalidade de orientar, esclarecer, encaminhar, transformar e conduzir o indivíduo a caminhos anteriormente não vislumbrados.

A educação propicia modificações no agir, nos valores, nas convicções, no enxergar o mundo do indivíduo (Santos et al., 2011). Nesse sentido, torna-se possível a transição do entrever de um mundo inerte, para o vislumbrar de uma realidade que se modifica, se renova e se recria, ou seja, a educação é um meio de formar indivíduos capazes de mudar não somente a sua realidade, mas também do mundo que está (Freire, 2014).
Portanto, estimular a educação é uma estratégia factível dentro do universo da saúde e em todas suas esferas de assistência, uma vez que essa necessita ser iniciada e continuamente renovada, visto a dinamicidade do viver. Todavia, as ações de educação em saúde ainda são desafiadoras, principalmente, no complexo ambiente hospitalar. Diante dessa realidade, a utilização de recursos que otimizem o processo de educação em saúde e estimule o preparo do paciente para a alta hospitalar representa uma estratégia na busca pela continuidade do tratamento.

A esse respeito, pode-se dizer que a alta hospitalar configura-se como um momento delicado e de grande ansiedade, havendo uma transição do cuidado profissional para o cuidado domiciliar, realizado tanto pelo paciente, quanto por seus familiares.

Objetivando melhor preparar o paciente para a alta hospitalar, Mendez et al. (2019), colocam que o uso de aplicativos móveis na área da saúde tem potencial para melhorar os resultados entre aqueles que vivem com doenças crônicas através do controle aprimorado dos fatores de risco, estimular a coparticipação do paciente no seu tratamento e a participação familiar, além de trazer um estímulo ao cuidado com a saúde.

Amstrong et al. (2017) abordam o uso da tecnologia em saúde como um olhar além da cura ou conforto da doença, mas sim como uma forma de integrar o indivíduo com todos os aspectos que fazem parte de seu viver. As autoras pontuam que o avanço do uso das tecnologias educativas em saúde avanço no desenvolvimento do uso de tecnologias móveis e comunicação em saúde tem sido considerado uma estratégia eficaz para incentivar os pacientes a adotar estilos de vida saudáveis, realizar a orientação sobre a doença, a fim de esclarecer pontos ainda obscuros de sua doença, estimular o autocuidado, reforçar as condições de doenças crônicas e diminuir o tempo e o custo para o paciente e o sistema de saúde.

Dentre as tecnologias educativas utilizadas na educação em saúde, a cartilha é entendida como um instrumento facilitador, uma vez que possibilita apresentar os conteúdos de uma forma lúdica e atrativa. Segundo Grippo e Fracolli (2008) a cartilha fomenta a discussão entre os profissionais, pacientes e familiares, gerando uma reflexão crítica acerca de sua atual realidade, fortalecendo, com isso, a autoconfiança do indivíduo. Assim fazendo, a cartilha caracteriza-se 
como um instrumento de saúde válido e de fácil compreensão, fato esse que auxilia na adesão ao tratamento proposto e, consequentemente, na construção de um pensar voltado a uma nova forma de viver.

\section{Conclusão}

Os resultados desse estudo apontam para a necessidade da realização de um melhor planejamento para alta hospitalar de pacientes com DAOP, de modo que o processo de educação em saúde perpasse por todas as etapas da internação hospitalar, tendo seu início no acolhimento do paciente e sua finitude através da contrarreferência.

Verificou-se que os pacientes diagnosticados com DAOP possuem pouco conhecimento acerca do seu diagnóstico e dos fatores de risco para o desenvolvimento e evolução da doença. Fato, tal, que contribui para o agravamento de sua condição de saúde, podendo ocasionar, em casos mais graves, mutilações e óbitos.

Identifica-se que o processo de educação em saúde tem sido uma atividade de pouco prestígio entre os profissionais da área, pois alguns pacientes relataram desconhecer completamente o seu diagnóstico. É possível que fatores como a sobrecarga de trabalho esteja relacionada com essa problemática, visto que o hospital configura-se com um ambiente de grande complexidade e altamente tecnológico, o que pode dificultar as relações humanas e a interação entre paciente e profissional. Entretanto, a tecnologia em saúde pode e deve ser utilizada com objetivo de facilitar o processo de educação em saúde e quando bem aplicada excelentes resultados são alcançados, sobretudo no que tange ao preparo do paciente para alta hospitalar.

O fato é que independentemente do caminho a ser seguido, faz-se necessário uma reflexão acerca de como o processo de educação em saúde vem sendo realizado entre as três esferas de assistência à saúde, pois entende-se que a promoção da saúde e a manutenção do bem-estar do paciente encontram-se diretamente relacionadas a conscientização dos fenômenos que anteveem o seu adoecimento, assim como das medidas necessárias para o defronte da doença.
Visualizar o paciente em sua integralidade e propor uma terapêutica holística e individualizada configura-se como uma necessidade dentro do setor saúde. Dessa forma, observou-se que a compreensão do paciente quanto à cronicidade de seu diagnóstico, o entendimento dos fatores de risco para o surgimento da doença, bem como a presença de uma rede de apoio social eficiente apresentam-se como elementos determinantes no êxito e na adesão a terapêutica prescrita.

\section{Contribuições das autoras}

Amante, L. N. participou da análise dos dados da pesquisa, interpretação dos resultados, redação do artigo científico e encaminhamento do artigo científico. Martins, T., Salum, N. C. e Locks, M. O. H. participaram da concepção, delineamento, busca e análise dos dados da pesquisa, interpretação dos resultados, redação do artigo científico. Girondi, J. B. R. e Sebold, L. F. participaram da interpretação dos resultados e redação do artigo científico.

\section{Conflitos de interesses}

Nenhum conflito financeiro, legal ou político envolvendo terceiros (governo, empresas e fundações privadas, etc.) foi declarado para nenhum aspecto do trabalho submetido (incluindo mas não limitando-se a subvenções e financiamentos, participação em conselho consultivo, desenho de estudo, preparação de manuscrito, análise estatística, etc.).

\section{Referências}

Alvim R.O., Dias, F.A.L., Oliveira C.M., Horimoto A.R.V.R., Ulbrich A.Z., Krieger J.E.,... \& Pereira A.C. (2018). Prevalência de doença arterial periférica. International Journal of Cardiovascular Sciences, 31(4), 405-413. Recuperado de http://www.scielo.br/scielo.php?pid=\$235956472018000400405\&script=sci_abstract\&tlng=pt. doi: 10.5935/2359-4802.20180031

Armstrong K.A.; Coyte P. C., Brown M., Beber B., \& Semple J. L. (2017). Effect of home monitoring via mobile app on the number of in-person visits following ambulatory surgery: a randomized clinical trial. JAMA Surg, 152(7),622-

7. Recuperado de https://www.ncbi.nlm.nih.gov/ pubmed/28329223. doi: 10.1001/jamasurg.2017.0111

Bastos, T.F, Alves, M.C.G.P., Barros, M.B.A., \& Cesar, C.L.G. (2012). Men's health: a population based study on social inequalities. Cadernos de Saúde Pública, 28(11),2133-2142. Recuperado de http://www.scielo.br/scielo.php?script=sci_ arttext\&pid=S0102-311X2012001100013 . doi: $10.1590 /$ S0102-311X2012001100013 
Barros, E.J.L., Santos, S.S.C., Gomes, G.C., \& Erdmann, A.L. (2012). Gerontotecnologia educativa voltada ao idoso estomizado à luz da complexidade. Revista Gaúcha de Enfermagem, 33(2), 95-101. Recuperado de http://www.scielo.br/scielo. php?script=sci_arttext\&pid=S1983-14472012000200014. doi: 10.1590/S1983-14472012000200014

Berto, S.J.P., Carvalhaes, M. A B.L., \& Moura, E.C.(2010). Tabagismo associado a outros fatores comportamentais de risco de doenças crônicas não transmissíveis. Cad Saúde Pública, 28(8),1573-1582. Recuperado de http://www. scielo.br/scielo.php?script=sci_arttext\&pid=S0102311X2010000800011. doi: 10.1590/S0102$311 \times 2010000800011$

Brondani, C.M, Beuter, M., Alvim, N.A.T., Szareski, C.,... Rocha, L.S. (2010). Cuidadores e estratégias no cuidado ao doente na internação domiciliar. Revista Texto \& Contexto Enfermagem, 19(3),504-510. Recuperado de http://www. scielo.br/scielo.php?script=sci_abstract\&pid=S0104$07072019000100301 \&$ lng=pt\&nrm=iso. doi: 10.1590/1980-265X-TCE-2017-0364

Caiafa J.S., Castro, A.A., Fidelis, C., Santos,V.P., Silva, E.S., \& Sitrângulo Jr C. (2011). Atenção integral ao portador de pé diabético. Jornal Vascular Brasileiro, 10(4), supl. 2, 1-32. Recuperdo de http://www.scielo.br/scielo.php?script=sci_ arttext\&pid=S1677-54492011000600001 doi: $10.1590 /$ S1677-54492011000600001

Diniz, J.N., Pires R.C.C.P. (2010). Percepção da doença arterial obstrutiva periférica por pacientes classe I ou II de Fontaine de um Programa de Saúde da Família. Jornal Vascular Brasileiro, 9(3),124-130. Recuperado de http://www.scielo.br/scielo.php?pid=S1677$54492010000300005 \&$ script=sci_abstract\&tlng=pt. doi: 10.1590/S1677-54492010000300005

Fonseca, C., Franco, T., Ramos, A., \& Silva, C. (2012). A pessoa com úlcera de perna, intervenção estruturada dos cuidados de enfermagem: revisão sistemática da literatura. Revista da Escola de Enfermagem USP, 46(2), 480-486. Recuperado de http://www.scielo.br/scielo.php?script=sci_ arttext\&pid=S0080-62342012000200029. doi: $10.1590 /$ S0080-62342012000200029

Freire, P. (2014). Pedagogia da Autonomia: saberes necessários à prática educativa (48. ed.). Rio de Janeiro: Paz e Terra.

Garofolo, L., Ferreira, S.R.G., \& Miranda Junior, F. (2014). Biomarcadores inflamatórios circulantes podem ser úteis para identificar doença arterial obstrutiva periférica mais grave. Jornal Vascular Brasileiro,13(3),182-191. Recuperado de http://www.scielo.br/scielo.php?script=sci_ arttext\&pid=S1677-54492014000300182. doi: $10.1590 /$ jvb.2014.045
Gerage, A.M., Correia, M. A., Oliveira, P. M. L., Palmeira, A. C. Domingues, W. J. R.,... Zeratti, A. E. (2019). Níveis de atividades físicas em pacientes com doença artetial periférica. Arquivos Brasileiros Cardiologia, 113(3), 410-416. Recuperado de http://www.scielo.br/scielo. php?pid=S0066-782X2019000900410\&script=sci_ abstract\&tIng=pt. doi: 10.5935/abc.20190142

Grippo, M.L.V.S., Fracolli, L.A. (2008). Avaliação de uma cartilha educativa de promoção ao cuidado da criança a partir da percepção da família sobre temas de saúde e cidadania. Revista da Escola de Enfermagem da USP, 42(3),430-6. Recuperado de http://www.scielo.br/scielo.php?script=sci_ arttext\&pid=S0080-62342008000300003. doi: $10.1590 /$ S0080-62342008000300003

Hospital Univesitário Professor Polydoro Ernani de São Thiago. Estruturas/ Setores. (2016). Recuperado de http://www. hu.ufsc.br/

Lopes, P.R., Barbosa, J.P.A.S., Lima, A.H.R.A., Miranda, A.S., Rodrigues, L.B.C.C., Rodrigues, S.L.C.,... Dias, R.M.R. (2012). Triagem pré-participação em exercício físico em pacientes com doença arterial periférica. Jornal Vascular Brasileiro, 11(3),194-198. Recuperado de http://www.scielo.br/ scielo.php?pid=S1677-54492012000300005\&script=sci_ abstract\&tlng=pt. doi: 10.1590/\$1677-54492012000300005

Makdisse, M., Pereira, A.C., Brasil, D.P., Borges, J.L., MachadoCoelho,G.L.L., Krieger, J.E.,...Neto, R.M.N. (2008). Prevalence and risk factors associated with peripheral arterial disease in the hearts of Brazil project. Arquivos Brasileiros de Cardiologia, 91(6),402-414. Recuperado de http://www.scielo.br/scielo.php?script=sci_ arttext\&pid=S0066-782X2008001800008. doi: $10.1590 /$ s0066-782X2008001800008

Malta, D.C; Merhy, E.E. (2010). O percurso da linha do cuidado sob a perspectiva das doenças crônicas não transmissíveis. Interface - Comunicação, Saúde, Educação, 14(34),593-606. Recuperado de http://www.scielo.br/ scielo.php?pid=S1414-32832010000300010\&script=sci_ abstract\&tlng=pt. doi: 10.1590/S1414-32832010005000010

Marinho, J.C.B., Silva, J.A. (2015). Concepções e implicações da aprendizagem no campo da educação em saúde. Ensaio Pesquisa em Educação e Ciência, 17(2), 351-371. Recuperado de http://www.scielo.br/scielo.php?pid=S1983$21172015000200351 \&$ script=sci_abstract\&tlng=pt. doi: 10.1590/1983-21172015170204

Mendez, C. B., Salum, N.C., Junkes, C., Amante, L.N., \& Mendez, C. M.L. (2019). Aplicativo móvel educativo e de follow up para pacientes com doença arterial periférica. Revista Latino-Americana de Enfermagem, 27:e3122. Recuperado de http://www.scielo.br/scielo.php?script=sci_ abstract\&pid=S0104-11692019000100306\&lng=en\&nrm=i so\&tlng=pt. doi: 10.1590/1518-8345.2693-3122 
Migowski, A., Chaves, R.B.M., Coeli, C.M., Ribeiro, A.L.P., Tura, B.R., \& Kuschnir, M.C.C. (2011). Acurácia do relacionamento probabilístico na avaliação da alta complexidade em cardiologia. Revista de Saúde Pública, 45(2), 269-275. Recuperado de http://www.scielo.br/scielo.php?script=sci_ arttext\&pid=S0034-89102011000200005. doi: $10.1590 /$ S0034-89102011005000012

Minayo, M. C. S. (2013). O Desafio do Conhecimento (12a ed.). São Paulo: Hucitec.

Ministério da Saúde (2015). Secretaria de Vigilância em Saúde. Departamento de Vigilância de Doenças e Agravos não Transmissíveis e Promoção da Saúde. Vigitel Brasil 2014: vigilância de fatores de risco e proteção para doenças crônicas por inquérito telefônico / Ministério da Saúde, Secretaria de Vigilância em Saúde, Departamento de Vigilância de Doenças e Agravos não Transmissíveis e Promoção da Saúde. Recuperado de https://bvsms.saude. gov.br/bvs/publicacoes/vigitel_brasil_2017_vigilancia_ fatores_riscos.pdf

Olin, J.W., White C.J., Armstrong, E.J., Kadian-Dodov. D., \& Hiatt, W.R. (2016). Peripheral artery disease: evolving role of exercise, medical therapy, and endovascular options. J Am Coll Cardiol, 67(11):1338-57. Recuperado de https:// www.ncbi.nlm.nih.gov/pubmed/26988957. doi: 10.1016/j. jacc.2015.12.049

Oliveira, D. C., Santos, M.V. R., Gomes, V. R., Sarinho, F.W., \& Novaes, M. (2013). Prevalência de adesão a fármacos antihipertensivos: registro de mundo real. Revista da Sociedade Brasileira de Clinica Medica,11(3):219-22. Recuperado de http://files.bvs.br/upload/S/1679-1010/2013/v11n3/a3766. pdf

Polit, D. F., \& Beck, C. T. (2011). Fundamentos de pesquisa em enfermagem: avaliação de evidências para a prática da enfermagem (7 ed.) Artmed.

Reis, P.E.O., Reis, I.F.O., Neves, D.Q., Matsumoto, P.K., Han, S.W., \& Borojevic, R. (2012). Isquemia de Membros Inferiores: perspectiva atual da terapia celular e terapia gênica. Revista Brasileira de Cardiologia, 25(4),330-339. Recuperado de http://www.onlineijcs.org/english/sumario/25/pdf/ v25n4a10.pdf

Reis, P.G.T.A., Nakakogue, C., Nakakogue, T., Nasr, A., TCBCPR., Tomasich F.D.S., TCBC-PR., Collaço, I.A.,... TCBC-PR. (2013). Orientações de alta: cartões padronizados ajudam na compreensão dos pacientes do pronto socorro? Revista do Colégio Brasileiro de Cirurgiões, 40(4),335-341. Recuperado de http://www.scielo.br/scielo.php?script=sci_ arttext\&pid=S0100-69912013000400014. doi: $10.1590 /$ S0100-69912013000400014
Rolim, D., Sampaio, S., Gonçalves-Dias, P., Almeida, P., AlmeidaLopes, J., \& Teixeira, J.F. (2015). Mortalidade depois da amputação. Angiologia e Cirurgia Vascular, 11(3),166170. Recuperado de https://www.sciencedirect.com/ science/article/pii/S1646706X15000725. doi: 10.1016/j. ancv.2015.06.001

Sales, A.T.N., Fregonezi, G.A.F., Silva, A.G.C.B., Ribeiro, C.T.D., Dourado-Junior, M.E.T., Sousa, A.G.P.,... Lavezzo Di, F.A. (2015). Identification of peripheral arterial disease in diabetic patients and its association with quality of life, physical activity and body composition. Jornal Vascular Brasileiro, 14(1), 46-54. Recuperado de http://www.scielo.br/scielo.php?script=sci_ arttext\&pid=S1677-54492015000100046. doi: $\underline{10.1590 / 1677-5449.20140043}$

Santos, Z.M.S.A., Caetano, J.A., \& Moreira, F.G.A. (2011). Atuação dos pais na prevenção da hipertensão arterial: uma tecnologia educativa em saúde. Ciência \& Saúde Coletiva, 16(11), 4385-4394. Recuperado de scielo.br/scielo.php?script=sci_abstract\&pid=S1413$81232011001200011 \& \operatorname{lng}=e n \& n r m=i s o \& t \mid n g=p t$. doi: 10.1590/S1413-81232011001200011

Santos, V.P., Alves, C.A.S., Lopes, C.F., \& Filho, J.S.A. (2013). Diferenças entre os gêneros em pacientes com isquemia crítica por doença arterial obstrutiva periférica. Jornal Vascular Brasileiro, 12(4),278- 283. Recuperado de http://www.scielo.br/scielo.php?pid=S167754492013005013053\&script=sci_arttext\&tlng=pt. doi: 10.1590/jvb.2013.053

Serra, C.G., Rodrigues, P.H.A. (2010). Avaliação da referência e contrarreferência no Programa Saúde da Família na Região Metropolitana do Rio de Janeiro (RJ, Brasil). Ciência \& Saúde Coletiva, 15(supl. 3), 3579-3586. Recuperado de http://www.scielo.br/scielo.php?pid=S1413$81232010000900033 \&$ script=sci_abstract\&tlng=pt. doi: 10.1590/S1413-81232010000900033

Silva, R.C.G., Giribela, C.R.G., Wolosker, N., \& ConsolimColombo, F.M. (2012). Limitação funcional e claudicação intermitente: impacto das medidas de pressão arterial. Arquivos Brasileiros de Cardiologia, 98(2),161166. Recuperado de http://www.scielo.br/scielo. php?pid=S0066-782X2012005000001\&script=sci_ abstract\&tlng=pt. doi: 10.1590/S0066-782X2012005000001

Tavares, R.S., Silva, D.M.G.V. (2013). A implicação do apoio social no viver de pessoas com hipertensão arterial. Revista Gaúcha de Enfermagem, 34(3), 14-21. Recuperado de http://www.scielo.br/scielo.php?script=sci_ arttext\&pid=S1983-14472013000300002. doi: $10.1590 /$ S1983-14472013000300002 
Teixeira, J.P.D.S., Rodrigues, M.C.S., \& Machado, V.B. (2012). Educação do paciente sobre regime terapêutico medicamentoso no processo de alta hospitalar: uma revisão integrativa. Revista Gaúcha de Enfermagem, 33(2),186-196. Recuperado de http://www.scielo.br/scielo. php?script=sci_arttext\&pid=S1983-14472012000200026. doi: $10.1590 / S 1983-14472012000200026$

Torres, A.G.M., Machado, E.G., Lopes, T.S., Gentile, P.C.; Vieira, A.C., Soares, L.G., Soares, G.P.,... Soares, P.S.L. (2012). Prevalência de alterações do índice tornozelobraço em indivíduos portadores assintomáticos de doença arterial obstrutiva periférica. Revista Brasileira de Cardiologia,25(2),87-93. Recuperado de https://pesquisa. bvsalud.org/portal/resource/pt/lil-629911

Vaz, C., Duarte, V.M., Santos, A.R., Valente, P.; Paúl, C., Bastos, R.... Clara Nogueira, C. (2013). Doença arterial periférica e qualidade de vida. Angiologia e Cirurgia Vascular, 9(1),1723. Recuperado de http://www.scielo.mec.pt/scielo. php?script=sci_arttext\&pid=S1646-706X2013000100003 